\title{
Educación Científica, Reflexiones y Propuestas desde los Feminismos
}

\author{
Scientific Education, reflections, and proposals from feminisms \\ Educação científica, reflexões e propostas de feminismos
}

\author{
Johanna Patricia Camacho-González ${ }^{10}$
}

Recibido: septiembre 2019

Aceptado: abril 2020

Para citar este artículo: Camacho-González, J.P. (2020). Educación Científica, Reflexiones y Propuestas desde los Feminismos. Revista Científica, 38(2), 190-200. https://doi.org/10.14483/23448350.15824

\section{Resumen}

Este artículo presenta algunas reflexiones y propuestas desde los feminismos para la investigación en el área de la didáctica de las ciencias. Se trata de una discusión sobre los principales aportes de los estudios de género que pueden contribuir favorablemente a la discusión y coyunturas actuales en relación con el rol de los sujetos en la educación científica con el propósito de avanzar hacia sociedades que superan la inequidad y avanzan hacia la justicia social. En primer lugar, se presentan vínculos entre la investigación de los estudios de géneros y la didáctica de las ciencias en los últimos años. Posteriormente, la discusión se focaliza en tres estratégicos que permiten articular y orientar la discusión actual en relación con una educación científica con perspectiva de género. Finalmente, se señalan algunas reflexiones finales con el ánimo de generar nuevas propuestas y proyecciones futuras.

Palabras clave: Género, Educación Científica, Feminismos, Enseñanza y aprendizaje de las Ciencias.

\begin{abstract}
This article presents some reflections and proposals from feminisms for research in the area of science education. This is a discussion about the main contributions of gender studies that can contribute favorably to the current discussion and situations in relation to the role of subjects in scientific education with the purpose of moving towards societies that overcome inequality and move towards social justice First, there are links between the research of gender studies and the teaching of science in recent years. Subsequently, the discussion focuses on three strategies that allow articulating and guiding the current discussion in relation to a scientific education with a gender perspective. Finally, some reflections are pointed out with the aim of generating new proposals and future projections.
\end{abstract}

Keywords: Gender, Scientific Education, Feminisms, Teaching and learning of Science.

1. Universidad de Chile. Santiago de Chile, Región Metropolitana. Chile. jpcamach@gmail.com 


\section{Resumo}

Este artigo apresenta algumas reflexões e propostas de feminismos para pesquisas na área de ensino de ciências. Trata-se de uma discussão sobre as principais contribuições dos estudos de gênero que podem contribuir favoravelmente para a discussão e as situações atuais em relação ao papel dos sujeitos na educação científica, com o objetivo de avançar em sociedades que superam as desigualdades e avançam em direção à justiça social Primeiro, há ligações entre a pesquisa de estudos de gênero e o ensino de ciências nos últimos anos. Posteriormente, a discussão enfoca três estratégias que permite articular e orientar a discussão atual em relação a uma educação científica com perspectiva de gênero. Finalmente, algumas reflexões finais são apontadas com o objetivo de gerar novas propostas e projeções futuras. Palavras-chaves: Gênero, Educação Científica, Feminismos, Ensino e aprendizagem de ciências.

\section{Introducción}

El movimiento nacional feminista desarrollado por las estudiantes universitarias en Chile durante el año 2018 impulso nuevos temas en relación a la visión patriarcal en la construcción de conocimiento y el sexismo en las prácticas cotidianas. Esta situación vivida intensamente durante los meses de movilización nacional, proponen grandes desafíos para la educación, puesto que exigen repensar la naturaleza del conocimiento, las prácticas pedagógicas, el currículo oculto, las representaciones sociales que tenemos como herencias culturales, entre otros aspectos, a fin de lograr una educación de calidad.

En este contexto resulta bastante relevante re-mirar la educación en general y la educación científica en particular como campos de reproducción del sistema que ha tomado como punto de partida una visión de ciencia tradicional y androcéntrica, que ha invisibilizado la producción científica femenina, ha limitado la participación de las mujeres en la actividad científica y ha reproducido estereotipos de género a través de las prácticas pedagógicas, lo que supone importantes consecuencias en la vida de los niños, las niñas y jóvenes que no encuentran en la educación científica una oportunidad para comprender, tomar decisiones y transformar su vida.

El propósito de este artículo, es contribuir al debate sobre cómo los aportes desde los feminismos pueden enriquecer la educación científica, para transformar los procesos de enseñanza - aprendizaje en el contexto escolar en oportunidades que contribuyan a la equidad y la justicia social.

\section{Feminismos y educación científica}

El aporte de los movimientos feministas, se orientan hacia la superación de la desigualdad de género, la opresión/dominación de las mujeres por los hombres y las diferencias sociales de manera más transversal en donde se incluye como preocupaciones la clase, raza, etnicidad, sexualidad, discapacidad, entre otras. Entendemos que hay un escenario amplio de escuelas de pensamiento, organizaciones, colectivos y grupos que se agrupan en relación a focos específicos que cambian y se desarrollan según los tiempos y contextos y por tanto, es complejo asumir una única mirada del feminismo y por esto, consideramos la denominación los feminismos.

La investigación de los feminismos no se ha preocupado solo por comprender cómo y por qué se forma y perpetuán las desigualdades, también ha considerado cómo lograr cambios a través de la reflexión de la práctica del conocimiento (praxis) y en ese sentido la vinculación con la educación científica emerge relevando como punto central lo personal, lo personal es político, la dimensión subjetiva y experiencial, especialmente de las mujeres, las cuales se reconoce que son moldeadas por prácticas y estructuras de poder característicos de la ciencia, la educación y la escuela.

La relación ciencia, género y educación científica cuenta con gran trayectoria investigativa (Meyer, 2010) a través de los estudios sociales de la ciencia, los estudios de género y los estudios en educación / pedagogía a tal punto que desde hace 
más de una década forman parte de las agendas internacionales estableciendo objetivos prioritarios y estratégicos en el desarrollo de la sociedad (ONU, 2000; UNESCO, 2009; Naciones Unidas, 2017). No obstante a este marco, dicha relación se ha considerado de manera paulatina como un área de investigación y formación, especialmente en Estados Unidos, España, Inglaterra, Noruega y Suecia (Camacho, 2013; Batista et al., 2012; Gallego y Camacho, 2015). Las experiencias e investigación en América Latina aún son muy bajas con respecto a los demás países y la producción de artículos científicos al respecto es escasa en comparación con otras temáticas de la educación científica (Scantlebury, 2012).

Según la revisión realizada por Camacho (2013), la relación ciencia - género ha hecho parte de la agenda educativa en distintos niveles, atendiendo a la propuesta de Risman and Davis (2013) se puede decir que durante las décadas 80’s y 90’s se empiezan a manifestar declaraciones de la importancia que tiene la perspectiva de género para la educación científica, en particular para dar cuenta de las diferencias en los rendimientos escolares de chicos y chicas; al respecto, se propusieron iniciativas a nivel estructural y políticas públicas principalmente enfocadas hacia la equidad y acceso de las mujeres en la educación (Scantlebury and Baker, 2007; Scantlebury, 2012).

A finales del Siglo XX además se empezaron a desarrollar investigaciones que buscaban determinar algunos factores explicativos de esta situación y que son relacionados con el sistema escolar. Investigaciones sobre el profesorado, las actitudes científicas, motivaciones hacia las ciencias, análisis de los currículos, análisis de las expectativas, vocaciones científicas, cuyos resultados manifestaron la necesidad de proponer diferentes estrategias para reestructurar la educación científica desde la diversidad y multiculturalidad. A partir del año 2000, varias investigaciones estuvieron orientadas a levantar perspectivas teóricas que permitieran comprender esta relación, en particular sustentadas desde visiones epistemológicas socioconstructivistas y feministas. En las investigaciones realizadas a partir del año 2010, se ha transitado más hacia un nivel simbólico, en relación al profesorado y se propone analizar con mayor énfasis sus creencias y además de la perspectiva de género, se tienen en cuenta otros aspectos socioculturales como: raza, etnia, religión, sexualidad, nivel socio económico, los que se detallan a través de estudios de caso o con pequeños grupos.

A partir de las contribuciones de los feminismos es posible centrar la discusión en los siguientes tres puntos:

\section{Re-significando la actividad científica y desenmarañando la tradición patriarcal}

Históricamente se ha demostrado que existe una visión androcéntrica y tradicional de la actividad científica, que supone entender la ciencia como una actividad objetiva, racional, inductiva, neutra y analítica (Camacho, 2013; 2017; Fernández et al.,1995; Longino, 1990; Lynch y Nowosenetz, 2009; Manassero y Vásquez, 2003), dominada por los hombres, en donde habitualmente se ha limitado e incluso invisibilizado la participación, la producción y el pensamiento de las mujeres (Schiebinger, 2004; Watts, 2007; Stadler, 2007; Buccheria et al., 2011). Sandra Harding (1986), Donna Haraway (1985) y Evelyn Fox-KeIler (1993) han cuestionado la naturaleza objetiva de la ciencia desde la perspectiva crítica feminista, ellas señalan que la hegemonía intelectual que existe es de orden masculina, una visión androcéntrica que tiene un trasfondo político cultural y conllevan a una

"imagen de ciencia distorsionada y empobrecida de la realidad que oculta las relaciones de poder y de posesión del orden simbólico masculino sobre las mujeres. Supone, además, la imposición de modelos únicos y arquetípicos de <<ser $>>$ : un único modelo masculino y un único modelo femenino, enfrentados por oposición (González y Lomas, 2006, 223). 
Es decir, que la ciencia como institución tradicionalmente sexista requiere un cuestionamiento interno como parte de su propia praxis que ofrezca una mirada crítica a sus particularidades, alejándose de una falsa neutralidad que ha caracterizado su contexto histórico y ha establecido una relación entre masculinidad y objetividad en su trabajo, aspecto que ha sido considerado en algunas publicaciones relevantes como el monográfico del día de la mujer de la Revista Nature (2013).

Analizar la actividad científica desde las miradas de los feminismos, supone comprender el género como una perspectiva transversal, una multiplicidad de intersecciones (culturales, demográficas, económicas, educativas, socioeconómicas, étnicas, políticas, raciales, sexuales, sociales) y no sólo una categoría biológica que define de manera homogénea dos grupos: hombres y mujeres (Exposito, 2012; Fausto Sterling, 2006); además de problematizar la propia naturaleza del conocimiento científico y sus mecanismos de producción, enseñanza, valoración y aplicación (Harding, 2012). Los aportes de la teoría crítica feminista de la ciencia permiten comprender la naturaleza de la ciencia de una manera dinámica, la relación de la actividad científica en contextos sociales, culturales y políticos, una visión holística, de heterogeneidad ontológica, de interacción mutua, aplicada a las necesidades, que resuelve problemas y de carácter explicativo, tal como lo señala la UNESCO (2009).

\section{La perspectiva de género en la Educación Científica es más que la participación de las mujeres}

Como señala Camacho (2018b), en Chile la educación científica desde sus orígenes hasta el día de hoy ha tensionado la perspectiva de género, tanto en la naturaleza de la ciencia y la tecnología que se enseña y aprende, como en los procesos de socialización que ocurren al interior del aula y de las instituciones. Esta tensión surge desde el momento en que se introduce el área científica en la educación, la cual, según M. Isabel Orellana (2012), estuvo condicionada por el género, ella afirma que en 1860 el plan de estudio de la Escuela Normal Femenina excluía la geometría y la química, disciplinas exclusivas para los varones; mientras que a las mujeres se les enseñaba economía doméstica, costura, bordado y "labores de aguja". Según esta misma autora, desde mediados del S. XIX las ciencias estuvieron presentes en los planes de estudio: en la educación primaria se enseñaba biología, botánica y zoología; y en la educación secundaria, se establecía con más fuerza la capacidad de observación, análisis y síntesis a través de la actividad experimental. Sin embargo, este último ciclo de profundización se reservaba para quienes querían seguir una carrera universitaria, que eran mayoritariamente hombres.

A fines del siglo XIX, a través del Decreto Amunátegui de 1877, se dio un gran impulso a la educación universitaria de las mujeres, especialmente para la emergente clase media. Fue así como a inicios del siglo XX, además de existir una red consolidada de liceos femeninos del Estado (que eran administrados de forma distinta que los liceos masculinos a través del Ministerio de Instrucción Pública) ya habían 17 mujeres con título universitario entre quienes figuraban dos abogadas, ocho profesoras de liceo, una farmacéutica y seis médicas cirujanas, entre ellas Eloísa Díaz Insunza, Ernestina Pérez Barahona y Eva Quezada Acharán, primeras con grado académico y título universitario de la Universidad de Chile y de Latinoamérica, quienes se ocuparon entre otros temas, por la salud pública femenina. A finales del siglo XX el número de mujeres universitarias alcanzaba a cuatrocientos veinte, siendo las profesoras y las farmacéuticas las más numerosas (Salas, 2016). Desde mediados del S. XX se han implementado varias iniciativas orientadas a aumentar la participación en los ámbitos científicos de las mujeres, de tal manera de lograr la alfabetización científica, la equidad de género y un mejor desarrollo científico y tecnológico. Estas iniciativas han ido desde la ampliación de la cobertura educativa para aumentar los 
niveles de educación formal de la población (Guerrero, et al., 2006), hasta fomentar políticas educativas orientadas a fortalecer el posicionamiento académico y el rol económico de las jóvenes en la educación terciaria y el sector productivo (Arcos, et al., 2007).

Sí bien se ha incrementado la participación de las mujeres en todas las áreas, contamos con una mujer ex Presidenta de la República y cuatro Premios Nacionales en las áreas científicas son mujeres (una en ciencias exactas y tres en ciencias naturales), distintos estudios evidencian la deuda histórica que existe con las mujeres, en cuanto ellas han sido poco visibles en comparación a los hombres como referentes de los aspectos científicos, políticos, económicos, sociales y culturales, puesto que a pesar de estas iniciativas aún persisten brechas de género, Chile está en el lugar 63 entre 144 naciones - del ranking del índice Global de Brecha de Género del Foro Económico Mundial (Global Gender Gap, 2017).

Según la encuesta CASEN (2015) la tasa neta de asistencia a la educación básica de hombres y mujeres es igual (91,1\%) y en la educación media es de $71,7 \%$ para hombres y $75,6 \%$ para mujeres. Este componente de género ha demostrado la dificultad que existe para el estudiantado chileno en general y para las chicas en particular, alcanzar los niveles de alfabetización científica esperados. Los resultados de PISA 2015 muestran puntajes promedios bajos respecto a los demás países OCDE (lugar 44 de 72) y además, indican que es el tercer país con mayor brecha negativa hacia las chicas tanto en los resultados de ciencias como en los de matemática (OCDE, 2016). Estos indicadores se pueden complementar además con la brecha que existe a favor de los hombres en los puntajes de la Prueba de Selección Universitaria (PSU) en las áreas de ciencias básicas, a pesar que las mujeres tienen mejor promedio las notas de enseñanza media (NEM) que los hombres (ellas 554 puntos, ellos 526 puntos, en el 2017) y por lo tanto, son gran parte de ellos $(47,2 \%)$ quienes ingresan a carreras de Tecnología (Ingenierías informática, electrónica y electricidad) a diferencia de las mujeres que ingresan a estas carreras (9,1\%). En las áreas de ciencias básicas (biología, química, matemática, física, astronomía, estadística y geología) el ingreso es bajo con relación a otras carreras (2\%) y las diferencias entre el ingreso de hombres y mujeres, se da en términos específicos, los hombres ingresan más a las ciencias físicas y las mujeres superan el $50 \%$ en las ciencias naturales.

Entonces, hombres y mujeres ingresan en la misma proporción al sistema educativo, en general el estudiantado chileno no alcanza los niveles esperados de alfabetización científica, en particular las chicas tienen menores resultados; las carreras de Tecnología son donde más ingresan estudiantes a nivel nacional, pero son las que tienen mayor desigualdad por sexo en la distribución de la matrícula y son masculinizadas, no solo en su composición sino además en su estructura (Cerón, 2018) y además, la matrícula en ciencias básicas es baja y mantiene estereotipos, en tanto que los chicos van a las carreras de ciencias físicas y las mujeres a las de ciencias naturales.

Con estos antecedentes se evidencia que el problema de la desigualdad en la educación científica no se resuelve con aumentar el acceso y la participación de las mujeres, por lo tanto, se propone evitar miradas reduccionistas de la perspectiva de género que la consideran como un factor o una variable definida por aspectos biológicos (sexo) y que junto con otras categorías sociales como variables permita explicar las brechas. En este sentido proponemos ampliar la mirada a una dimensión epistemológica y pedagógica, que permitan comprender la complejidad de los procesos educativos a través de niveles dinámicos y cambiantes que resignifican lo que es "femenino" y "masculino" como construcciones sociales.

\section{Desafíos para una educación científica que promueva la equidad y la justicia social}

Desde finales del siglo pasado y durante la primera década del Siglo XXI hay consenso general 
en asumir que la ciencia ocupa un rol importante en el desarrollo sostenible de la sociedad. Por lo tanto, se considera que el conocimiento científico transciende los aspectos cotidianos y hace parte de la cultura, es decir que la educación científica asume un objetivo social prioritario que aporta a la inclusión y la equidad social (Macedo y Katzkowicz, 2005) en un sentido amplio, que abarca todos los niveles y modalidades del sistema escolar y que está orientada a que las personas sean capaces de usar el conocimiento científico con el fin de entender y ayudar a tomar decisiones sobre el mundo natural y los cambios realizados en él a través de la actividad humana (Harlen 2002), logrando la alfabetización científica.

La educación científica según los términos anteriores se relaciona de manera coherente con la perspectiva de género bajo un enfoque denominado sensible o coeducativo, que incluye propuestas a partir de la Teoría Crítica Feminista (Roser, 1990; Harding, 1996; Fox-Keller, 1993), ya que reconoce la formación integral de las personas y asume que las categorías asociadas a los sexos no son binarias, ni homogéneas en tanto incluyen elementos de la cultura y por lo tanto, no interpretan el mundo de la misma manera, así comprenden la ciencia como un conocimiento situado, todas las historias incluyendo historias científicas, son funciones de la política y la contextualización de la persona que realiza la investigación, es imposible tener una única verdad. Así, la o el estudiante se entiende como persona diversa que construye su identidad, la cual también es parcialmente definida y limitada por otros aspectos como la clase social, la raza, etnia, cultura, lenguaje entre otros.

Esto supone integrar explícitamente en la enseñanza - aprendizaje de ciencias en el contexto escolar, las dimensiones sociales, políticas y culturales de la actividad científica; la incorporación de otros sistemas de conocimiento; así como los diferentes puntos de vista que existen en las ciencias; materiales educativos que incluyan el desarrollo científico de minorías y otras culturas; visualizar en la sala de clase que la actividad científica tiene una dimensión humana y que esto influye su desarrollo, además de promover las actividades en grupos mixtos con distintos intereses (Camacho, 2018b; Sinnes, 2006).

El rol del profesorado de ciencias es esencial para lograr una educación científica para la equidad y la justicia social, ya que se ha demostrado que a través del discurso y quehacer profesional de manera consciente o no, influye en los aprendizajes científicos (Catalán et al., 2011; Cabezas, 2010; Scantlebury y Martin, 2010; Scantlebury y Baker, 2007), en la motivación y los intereses del estudiantado (Chiu, 2010; Uitto et al., 2011), así como en la percepción que puede tener el estudiantado con respecto a la aplicabilidad de las ciencias en la vida cotidiana (Brickhouse et al., 2000) y los estereotipos de género en el aula (Fernández et al., 1995; Bianchini et al., 2000; Izquierdo, García y Solsona, 2009; Bucheria et al., 2011; Krapp y Prenzel, 2011). Según otras investigaciones, las/los docentes cumplen una función fundamental en los procesos de socialización en el aula (Barber y Mousher, 2007; Labudde et al., 2000; Sernam, 2009; OCDE, 2012), por lo que se consideran un aspecto clave para mejorar la relación ciencia-género en la educación científica y por ello, se deben estudiar sus creencias y prácticas como aspectos fundamentales, para comprender el aula como un espacio socio cultural donde se construyen y discuten conocimientos escolares (OEI, 2008).

Según Chetcuti (2009) la/el docente es un sujeto que trae consigo al aula, una compleja red de experiencias, habilidades, conocimientos, perspectivas e intereses, incluida su propia experiencia, su identidad de género, así como sus expectativas frente a su estudiantado. Desde allí, se posiciona frente a las relaciones ciencia-género y establece modos de interacción y comunicación con sus estudiantes (Gray y Leith, 2004). Estas creencias se desarrollan a través de la socialización y hacen parte de una cultura heredada (Murphy y Whitelegg, 2006), que, según Duarte et al., (2010) reflejan 
lo que sucede en la sociedad y los estereotipos que existen acerca de la ciencia y el género.

Esta compleja configuración del profesorado como sujetos, no es condiciona por su sexo, no por ser mujer es necesariamente feminista, ya que se ha hecho énfasis en la noción de sexo invariante, en cuanto que sus creencias y prácticas están relacionadas con sus experiencias y su propia visión de lo qué es la ciencia, los propósitos de la educación y cómo configura a través de las experiencias personales, los procesos formativos y la práctica pedagógica su ejercicio docente y su reflexión sobre los propósitos de la enseñanza-aprendizaje de las ciencias en el contexto escolar.

Ahora bien, si prevalece una visión androcéntrica de la ciencia sustentada en la concepción más tradicional (objetiva, racional, inductiva, individual, neutral, experimental, analítica y competitiva) es altamente predecible que se proyecte una imagen estereotipada masculina en donde, los aspectos relacionados con el contexto valórico, social y cultural (heterogeneidad ontológica, interacción mutua, aplicabilidad a las necesidades, contextualización) y asociados con lo femenino son menos reconocidos, puesto que existe una barrera "objetiva" que no da cabida a la mirada de la ciencias desde los feminismos ya que supone implícitamente que la perspectiva de género no impacta la construcción de conocimiento porque la ciencia se sustenta en sí misma como un cúmulo de contenidos descontextualizados, ahistóricos, que no hacen parte de la cultura y que además pueden ser pasados en las clases "pasar la materia".

Este Modelo de Ciencia Neutral, enmascara el patriarcado como igualdad entre hombres y mujeres al suponer que todos pueden construir el mismo conocimiento científico, siempre y cuando tenga el mismo rigor y objetividad y así se justifica que las mujeres/niñas deben esforzarse más porque históricamente han estado en una situación de desventaja; se omiten alusiones al sexo o roles de hombres y mujeres a través del currículo, los materiales y textos escolares (uso de lenguaje genérico masculino; noción de cuerpo asexuado o binario, blanco y heterosexual; imágenes o fotografías mayoritariamente de hombres científicos); se generan interacciones diferenciadas que suponen más atención y mayor complejidad para quienes están más interesados o con quienes se puede desarrollar mejor el contenido (en general varones); se estereotipan los roles de los hombres y las mujeres (el hombre de ciencia, la esposa de un científico, las chicas son más ordenadas escriben los informes, los chicos son más concretos experimentan); los ejemplos cotidianos estereotipados y/o que responden a intereses concretos sesgados (la fuerza como noción física relacionada como característica de hombres; referencia amplia de la ciencia experimental -instrumentos, aparatos que no se relacionan con el trabajo científico que realizan personas de manera grupal) y, se perpetúa una visión de ciencia en la escuela que además que no genera aprendizajes tampoco favorece la comprensión, la toma de decisiones y la transformación de la vida de los/las estudiantes y su entorno, es decir que la sala de clases de ciencias se convierte en un espacio de injusticia social (Iber, 2006).

\section{Conclusiones}

En ese sentido los feminismos y la investigación en didáctica de la ciencias relacionada, han generado algunas orientaciones para mostrar cómo la ciencia hace parte de la cultura y es una actividad socialmente construida y contextualizada y por tanto, la enseñanza - aprendizaje de las ciencias en el contexto escolar busca desarrollar y promover nuevas formas más equitativas e inclusivas a través de los enfoques pedagógicos, la formación del profesorado, el currículo y los materiales educativos.

Algunas propuestas concretas iníciales para transformar los procesos de enseñanza-aprendizaje de las ciencias en la escuela, surgen con la necesidad de visibilizar las mujeres científicas, sus saberes y aportes a la ciencia en acuerdo 
con Camacho (2015) y Álvarez, Ñuño y Solsona (2003), estas actividades pueden ser: a) elaboración de biografías de mujeres científicas - deseable que sean de Chile, vivas y activas - para que el estudiantado conozca modelos de personas de ciencias más cercanos y menos estereotipados; b) discusión del contexto socio-político y aspectos culturales para reflexionar acerca de cómo se construye el conocimiento científico -identificando obstáculos, errores, tensiones - para evitar la mirada triunfalista y exitista de la ciencia; c) incluir intencionadamente en las secuencias de enseñanza-aprendizaje textos o artículos con investigaciones, para dar a conocer los aportes de las mujeres, sus metodologías de trabajo y conclusiones; d) elaborar historietas acerca de las discusiones y los problemas que se desean resolver para construir explicaciones desde distintas miradas.

Esperamos que a partir del mayo feminista del 2018 se abran espacios de discusión, reflexión y formación que hasta el momento habían sido escasos en la formación inicial del profesorado de ciencias (Jara y Camacho, 2015), en un currículo nacional que en los últimos años ha intentado superar una visión tradicional de ciencia incluyendo aspectos históricos, sociales y culturales, pero que tímidamente propone estrategias concretas que asuma visiones sociocríticas y feministas de la actividad científica y, en un escenario nacional, que conoce la problemática y busca empoderar a las mujeres, pero que reclamará mecanismos de acción para iniciar cambios profundos.

\section{Agradecimientos}

Artículo Financiado por el Proyecto FONDECYT Regular 1201229

\section{Referencias}

Álvarez, M., Ñuño, T., Solsona, N. (2003). Las científicas y su historia en el aula. Madrid, España: Síntesis Educación.
Arcos, E., Figueroa, V., Miranda, C., Ramos, C. (2007). Estado del arte y fundamentos para la construcción de indicadores de género en educación. Estudios pedagógicos (Valdivia), 33(2), 121-130.

Barber, M., Mourshed, M. (2007). How the World's Best-Performing School Systems Come Out On Top. www.mckinsey.com

Batista, I. D. L., Torejani, A., Heerdt, B., Lucas, L. B., Ohira, M., Corrêa, M. L., ... Bastos, V. (2011). Gênero feminino e formação de professores na pesquisa em educação científica e matemática no Brasil. In Segunda Conferencia Latinoamericana del International History, Philosophy and Science Teaching Group.

Bianchini, J.A., Cavazos, L.M., Helms, J.V. (2000). From professional lives to inclusive practice: science teacher and scientists'views of gender and ethnicity in science education. Journal of Research In Science Teaching, 37(5), 511-547. https:// doi.org/10.1002/1098-2736(200008)37:6\%3C511::AID-TEA2\%3E3.0.CO;2-3

Brickhouse, N.; Lowery, P., Schultz, K. (2000). What kind of girls does science? The construction of school science identities. Journal of Research in Science Teaching, 37(5), 441-458. https://doi. org/10.1002/(SICI)1098-2736(200005)37:5\%3C441::AID-TEA4\%3E3.0.CO;2-3

Buccheri, G., Gürber, N. A., Brühwiler, C. (2011). The Impact of Gender on Interest in Science Topics and the Choice of Scientific and Technical Vocations. International Journal of Science Education, 33(1), 159-178. https://doi.org/10. $\underline{1080 / 09500693.2010 .518643}$

Cabezas, V. (2010). Gender peer effects in school: Does the gender of school peer affect student achievement? (PhD. Thesis). Columbia University.

Camacho, J. (2018a). Educación cientifica no sexista. Aportes desde la investigación en didáctica de las ciencias. Nomadías, (25), 101-120. https://nomadias.uchile.cl/index.php/NO/ $\underline{\text { article/view/51508 }}$ 
Camacho, J. (2018b). Percepción Social de la Ciencia y la Tecnología: una mirada desde la perspectiva de género. En: Garretón, M.A., Van den Eynde, A.M., Arancibia, M., Camacho, J., Molina, R.R. y Polino, C. Ciudadanía, Ciencia y Tecnología. Santiago, Chile. Comisión Nacional de Ciencia y Tecnología (CONICYT), 208-258.

Camacho, J. (2017). Identificación y caracterización de las creencias de docentes hombres y mujeres acerca de la relación ciencia - género en la educación científica. Estudios Pedagógicos, 43, 63-81. https://doi.org/10.4067/ $\underline{\mathrm{S} 0718-07052017000300004}$

Camacho, J. (2015). Algunos aportes de las mujeres a la cristalografía. Revista Educación en la Química. 21(1), 32-41

Camacho, J. (2013). Concepciones sobre ciencia y género en el profesorado de química: aproximaciones desde un estudio colectivo de casos. Ciência y Educação, 19(2013), 323-338. https:// doi.org/10.1590/S1516-73132013000200007

Catalán, M.A., García, R., Piedra, J., Vega, L. (2011). Diagnóstico de la cultura de género en educación: actitudes del profesorado hacia la igualdad. Revista de Educación, 355, 521-546.

Chetcuti (2009). Identifying a gender - inclusive pedagogy from Maltese science teachers'personal practical knowledge. International Journal of Science Education, 31(1), 81-99. https:// doi.org/10.1080/09500690701647996

Chiu, M. S. (2010). Effects of science interest and environmental responsibility on science aspiration and achievement: gender differences and cultural supports. Educational Research and Evaluation, 16 (4), 345-370. https://doi. org/10.1080/13803611.2010.527756

Duarte, C. (Coord). (2010). Representaciones sociales de Género, Generación e interculturalidad en textos escolares chilenos. Informe Final. Universidad de Chile, Facultad de Ciencias Sociales, Departamento de Sociología. http:// www.textosescolares.cl/usuarios/tescolares/
File/Informe\%20Final $\% 20$ Consolidado $\% 20$ 4 01'10.pdf

Einarsson, C., Granstrom, K. (2002) Gender-biased Interaction in the Classroom: the influence of gender and age in the relationship between teacher and pupil. Scandinavian Journal of Educational Research, 46(2), 117-127. https:// doi.org/10.1080/00313830220142155

Expósito, C. (2012). ¿Qué es eso de la interseccionalidad? Aproximación al tratamiento de la diversidad desde la perspectiva de género en España. Investigaciones Feministas, 3, 203-222. https:// doi.org/10.5209/rev INFE.2012.v3.41146

Fausto-Sterling, A. (2006) Cuerpos sexuados. La política de género y la construcción de la sexualidad. Barcelona: Melusina.

Fernández, C., Porta, I., Rodríguez, M., Solsona, N., Tarín, R. (1995). Una mirada no sexista a la clase de ciencias experimentales. Barcelona: Institut de Ciénces de l’Educación.

Fox-Keller, E. Reflexiones sobre género y ciencia. Valencia: Alfons el Magnànim. 1993

Gallego, A.P., Camacho, J. (2015). Género, Ciencia e Ingeniería: Un problema sociocultural (Editorial). Revista Científica, 25(1), 5-6. https://doi.org/10.14483/23448350.9650

González, A., Lomas, C. (Coords). (2006). Mujer y educación. Educar para la igualdad, educar desde la diferencia. Barcelona: Editorial Grao.

Gray, C., Leith, H. (2004) Perpetuating Gender Stereotypes in the classroom: a teacher perspective. Educational Studies, 30(1), 3-17. https:// doi.org/10.1080/0305569032000159705

Guerrero, E., Provoste, P., Valdés, A. (2006). Acceso a la educación y socialización de Género en un contexto de reformas educativas. En: Equidad de Género y Reformas Educativas Santiago, Chile: Hexagrama, 99-150.

Haraway, D. (2004). Testigo_Modesto@Segundo_ Milenio.hombrehembra@_Conoce_Oncoratón® Barcelona: UOC.

Harding, S. (2012). ¿Una filosofía de la ciencia socialmente relevante? Argumentos en torno a la controversia sobre el Punto de vista feminista. En: 
Blazquez, N., Flores, F., Ríos, M. Investigación Feminista. Epistemología, metodología y representaciones sociales. Centro de Investigaciones Interdisciplinares en Ciencias y Humanidades. Universidad Nacional Autónoma de México.

Harding, S. (1996). Ciencia y feminismo. Madrid. Ediciones Morata.

Izquierdo, M., García, C., Solsona, N. (2009). Géner $i$ ensenyament de les ciénces: representaciones $i$ propostes. Bellatera: Universitat Autónoma de Barcelona.

Jara, N., Camacho, J. (2015). Creencias sobre ciencia - género en la educación científica. Análisis de un estudio de caso en la formación inicial docente. Revista Educação \& Políticas em Debate, 4(2), 344-361

Krapp, A., Prenzel, M. (2011). Research on Interest in Science: Theories, methods, and findings. International Journal of Science Education, 33(1), 27-50. https://doi.org/10.1080/095006 $\underline{93.2010 .518645}$

Labudde, P., Herzog, W., Neuenschwander, M. P., Violi, E., Gerber, C. (2000). Girls and physics: Teaching and learning strategies tested by classroom intervention in grade 11 . International Journal of Science Education, 22(2), 143-157. https://doi.org/10.1080/095006900289921

Longino, H. (1990). Science as social knowledge: values and objetivity in Scientific inquiry. Pricenton: Princenton University Press.

López-Rivera, Z. C. (2015). La Enseñanza de las Ciencias Naturales desde el enfoque de la Apropiación Social de la Ciencia, la Tecnología y la Innovación ASCTI en la educación básica-media. Revista Científica, 2(22), 75-84. https://doi.org/10.14483/10.14483/udistrital. jour.RC.2015.22.a6

Lych, I., Nowosenetz, T. (2009). An exploratory study of students' constructions of gender in science, engineering and technology". Gender and Education, 21, 567-581.

Macedo, B., Katzkowicz, R. (2005). ¿Cómo promover el interés por la cultura científica? OREALC / UNESCO Santiago.
Mannasero, M.A., Vásquez, A. (2003). Los estudios de género y la enseñanza de las ciencias. Revista Educación, (330), 251-280.

Meyer, E. (2010). Gender and Sexual Diversity in Schools. Dordrecht, The Netherlands: Springer. https://doi.org/10.1007/978-90-481-8559-7

Murphy, P., Whitelegg, E. (2006). Girls and physics: Continuing barriers to 'belonging'. The Curriculum Journal, 17(3), 281-305. https://doi. org/10.1080/09585170600909753

Naciones Unidas. (2017) Objetivos de desarrollo sostenible (ODS) de la Agenda 2030 para el Desarrollo Sostenible. http:// www.un.org/sustainabledevelopment/es/ la-agenda-de-desarrollo-sostenible/

Orellana, M. I. (2012). Educación: Improntas de Mujer Serie Itinerario y memoria de Bicentenario. Santiago de Chile:Archivo Visual del Museo de Educación Gabriela Mistral. 2012

Organización de las Naciones Unidad para la Educación, la Ciencia y la Cultura (UNESCO) (2009). Aportes para la enseñanza de las ciencias del SERCE. Santiago, Chile.

Organización para la Cooperación y el DesarroIlo Económico (OECD) (2012). Resultados de PISA 2012 en Foco Lo que los alumnos saben a los 15 años de edad y lo que pueden hacer con lo que saben. Extraído de: https://www. oecd.org/pisa/keyfindings/PISA2012_Overview_ESP-FINAL.pdf

Risman, B., Davis, G. (2013). From sexrolestogender structure. Current Sociology, 61(5-6), 733-755. https://doi.org/10.1177/0011392113479315

Salas Neumann, E. (2006). Las mujeres chilenas que recibieron el siglo XX y las que lo despidieron.Santiago, Chile: Productora Gráfica Andros. 2006.

Sánchez-Robayo, B. J., Torres-Duarte, J. (2016). Aprender a investigar investigando. Realización de una propuesta de formación. Revista Científica, 1(28), 17-31. https://doi. org/10.14483/udistrital.jour.RC.2017.28.a2

Scantlebury, K., Baker, D. (2007). Gender issues in science education research: Remembering where the difference lies. In S. Abell \& N. 
Lederman (Eds.), Handbook of Research on Science Education. Mahwah, NJ: Lawrence Erlbaum. 2007, 257 -286

Scantlebury, K., Martin, S. (2010). How does she know? Re-visioning conceptual change from feminist perspectives". In W. Roth (Ed.), Re/ Structuring Science Education: Reuniting Sociological and Psychological Perspectives. Rotterdam: Springer. 173-186. https://doi. org/10.1163/9789460910869

Scantlebury, K. (2012). Still part of the conversation: Gender issues in Science Education. In B. Fraser, T. Kenneth, and M. Campbell (Eds.), Second International Handbook of Science Education, Dordrecht: Springer, 499-512. https:// doi.org/10.1007/978-1-4020-9041-7 34

Schiebinger, L. (2004). ¿Tiene sexo la mente? Valencia: Ediciones Cátedra.

Servicio Nacional de la Mujer (Sernam) (2009). Análisis del género en el aula. Documento de Trabajo 117. Santiago, Chile. Sernam.

Sinnes, A. (2006). Three approaches to gender equity in science education. Nordic Studies in
Science Education Nordina, 20(3), 72-83. https://doi.org/10.5617/nordina.451

Solbes-Matarredona, J., Torres-Merchán, N. Y. (2015). Alternativas para reflexionar aspectos críticos de la ciencia en el aula. Revista Científica, 2(22), 3144. https://doi.org/10.14483/10.14483/udistrital. jour.RC.2015.22.a3

Stadler, H. (2007). De-Constructing gender in science education. Cultural Studies of Science Education, 2, 68-979.

Uitto, A., Juuti, K., Lavonen, J., Byman, R., Meisalo, V. (2011). Secondary school students' interests, attitudes and values concerning school science related to environmental issues in Finland. Environmental Education Research, 17(2), 167-186. https://doi.org/10.1080/13504622.2010.522703

United Nations (ONU) (2000). Goals, targets and indicators! http://www.unmillenniumproject. org/goals/gti.htm\#goal3

Watts, R. (2007). Whose knowledge? Gender, education, science and history. History of education, 36(3), 283-302. https://doi. org/10.1080/00467600701279088 